\title{
Multiscale imaging of colitis in mice using confocal laser endomicroscopy, light-sheet fluorescence microscopy, and magnetic resonance imaging
}

Tianmeng Li

Hui Hui

Chaoen $\mathrm{Hu}$

$\mathrm{He} \mathrm{Ma}$

Xin Yang

Jie Tian 


\title{
Multiscale imaging of colitis in mice using confocal laser endomicroscopy, light-sheet fluorescence microscopy, and magnetic resonance imaging
}

\author{
Tianmeng Li, ${ }^{a, b, c, \dagger}$ Hui Hui, ${ }^{b, c, d,+}$ Chaoen Hu, ${ }^{b, c}$ He Ma, ${ }^{a, *}$ Xin Yang, ${ }^{b, c, *}$ and Jie Tian ${ }^{b, c, d, *}$ \\ ${ }^{a}$ Northeastern University, Sino-Dutch Biomedical and Information Engineering School, Shenyang, China \\ ${ }^{\mathrm{b} C h i n e s e}$ Academy of Sciences, Institute of Automation, CAS Key Laboratory of Molecular Imaging, Beijing, China \\ 'Institute of Automation, Beijing Key Laboratory of Molecular Imaging, Beijing, China \\ dUniversity of Chinese Academy of Sciences, Beijing, China
}

\begin{abstract}
The objective of our study is to develop a multimodality approach by combining magnetic resonance imaging (MRI) and optical imaging methods to assess acute murine colitis at the macro- and microscopic level. In vivo MRI is used to measure the cross-sectional areas of colons at the macroscopic level. Dual-color confocal laser endomicroscopy (CLE) allows in vivo examination of the fluorescently labeled epithelial cells and microvessels in the mucosa with a spatial resolution of $\sim 1.4 \mu \mathrm{m}$ during ongoing endoscopy. To further validate the structural changes of the colons in three-dimensions, ex vivo light-sheet fluorescence microscopy (LSFM) is applied for in-toto imaging of cleared colon sections. MRI, LSFM, and CLE findings are significantly correlated with histological scoring $(p<0.01)$ and the inflammation-associated activity index $(p<0.01)$. Our multimodality imaging technique permits visualization of mucosa in colitis at different scales, which can enhance our understanding of the pathogenesis of inflammatory bowel diseases. $\odot$ The Authors. Published by SPIE under a Creative Commons Attribution 4.0 Unported License. Distribution or reproduction of this work in whole or in part requires full attribution of the original publication, including its DOI. [DOI: 10.1117/1.JBO.24.1.016003]
\end{abstract}

Keywords: multimodality imaging; confocal laser endomicroscopy; light-sheet fluorescence microscopy; magnetic resonance imaging; inflammatory bowel disease.

Paper 180551RR received Sep. 30, 2018; accepted for publication Jan. 15, 2019; published online Jan. 30, 2019.

\section{Introduction}

Inflammatory bowel diseases (IBDs) are chronic inflammatory diseases with intestinal immune disorders, including Crohn's disease (CD) and ulcerative colitis (UC). ${ }^{1}$ Patients with IBDs show an increased risk for developing colorectal cancer (CRC), one of the leading causes of death worldwide. ${ }^{2}$ In the last decade, new imaging techniques have been developed for early detection of inflammatory lesions, which has resulted in a significant reduction in the incidences of CRC. ${ }^{3-6}$ However, the etiology of IBDs remains unclear due to its heterogeneous disorders with various appearances. In the previous study, the inflammation of UC has been mainly observed in the mucosal and submucosal layers; however, in $\mathrm{CD}$, the inflammation has been scattered and may be found in all the layers of colon. ${ }^{7}$ Therefore, to enhance our understanding of the pathogenesis of IBDs, powerful multimodality imaging techniques that can assess the colon at the macro- and microscopic level should be developed.

Macroscopic imaging techniques, such as magnetic resonance imaging (MRI), are widely used to evaluate the luminal alternations of colitis. ${ }^{8,9}$ MRI is preferable because it does not have the ionizing radiation emitted by computed tomography (CT) enterography. ${ }^{10}$ However, conventional MRI has too low a resolution to detect mucosal changes at the cellular level. An imaging method, confocal laser endomicroscopy (CLE), provides over 10-fold higher resolution than MRI for in vivo tracking of fluorescently labeled epithelial cells and microvessels

*Address all correspondence to $\mathrm{He} \mathrm{Ma}$, E-mail: mahe@bmie.neu.edu.cn; Xin Yang, E-mail: xin.yang@ia.ac.cn; Jie Tian, E-mail: jie.tian@ia.ac.cn

†These authors contributed equally to this work. of the mucosa. ${ }^{3}$ In a CLE system, thousands of thin fibers are incorporated in a probe, in which each fiber works as a point scanner and pinhole used in a conventional confocal microscope. ${ }^{11}$ In contrast to standard white-light endoscopy, the CLE system has real-time imaging of the mucosal alterations via the administration of fluorescent contrast agents to stain cells and vessels in the mucosa. ${ }^{12}$ The lateral resolution of CLE for mouse applications can reach up to $1.4 \mu \mathrm{m}$ with $10-\mu \mathrm{m}$ optical sectioning and a field of view (FOV) diameter from 240 to $600 \mu \mathrm{m}$.

Although CLE provides accurate assessment of mucosa, whole colon section imaging in three-dimensions (3-D) is still challenging due to its small FOV. Advanced imaging techniques, such as light-sheet fluorescence microscopy (LSFM) (also known as ultramicroscopy), allow for in-toto imaging of the fluorescently labeled transparent sample. ${ }^{13-16}$ Candeo et al. ${ }^{17}$ have developed a virtual unfolding of LSFM dataset for quantitative analysis of the murine intestine. Gabanyi et al. ${ }^{18}$ have used intravital multiphoton microscopy to monitor cell dynamics in different layers of the intestinal wall of live animals, and a 3-D view of macrophage distribution in the small intestine has been visualized by light-sheet microscopy. The aim of this study was to compare and correlate MRI, CLE, and LSFM to analyze their potential as multimodality tools to assess a chemically induced experimental model of IBDs at the macro- and microscopic level.

\section{Materials and Methods}

\subsection{Induction of Colitis Model}

All animal experiments were approved in accordance with the guidelines of the Institutional Animal Care and Use Committee 
(IACUC) of Peking University, and all procedures were performed in accordance with the approved guidelines of IACUC of Peking University. Male C57BL/6 mice $(n=15)$, six weeks old (Beijing Vital River Laboratory Animal Technology Co., Ltd.), were kept under standard housing conditions providing water and food ad libitum. To induce colitis, mice received 3.5\% weight/volume dextran sodium sulfate (DSS, 36 to $50 \mathrm{kDa}$, MP Biomedicals) in their drinking water for three days $(n=5)$ and six days $(n=5)$. Five mice were not treated with DSS and were used as a control group. DSS-induced model development was monitored with weight loss, diarrhea, and stool bleeding. ${ }^{19-21}$ Prior to MRI and CLE, mice were fasted for $12 \mathrm{~h}$.

\subsection{Magnetic Resonance Imaging Assessment}

MRI was performed on a 1-Tesla permanent magnet small animal scanner (M3TM Aspect Imaging, Israel). To assess the anatomical changes in the mice with IBDs, we used the T1weighted sequence (TR: $500 \mathrm{~ms}$, TE: $12 \mathrm{~ms}, 195 \mu \mathrm{m}$ in plane resolution, flip angle: $90 \mathrm{deg}$, acquisition matrix: $154 \times 154$, number of averages: 7 , slice thickness: $1 \mathrm{~mm}$, and duration: $10 \mathrm{~min}$ and $5 \mathrm{~s}$ ). MRI was started at day zero and repeated on day three and six after DSS induction. For MRI, mice were anesthetized with $1.5 \%$ isoflurane. Animals were kept on a water tube heating pad to keep the body temperature constant. Measurement of the diameter and thickness of the colons was performed in the ImageJ package FIJI (version $1.51) .^{22}$

\subsection{Confocal Laser Endomicroscopy Examination}

Prior to CLE (CellVizio Dual Band, Mauna Kea Technologies, France) examination, the mice colon vasculature was stained via intravenous injection with $100 \mu \mathrm{L} 2 \%$ weight/volume Evans blue (MedChemExpress). Subsequently, $100 \mu \mathrm{L}$ of $0.05 \%$ weight/volume acriflavine (Sigma-Aldrich) was administered topically for mucosa cell staining. ${ }^{23}$ Approximately 15 to $20 \mathrm{~min}$ after administering the dyes, CLE was performed by placing a fiber optic probe against the distal colon mucosa of each mouse. The CellVizio laser was used for confocal imaging; it generates excitation at both 488 and $660 \mathrm{~nm}$ and couples a dual-laser beam into the probe with $2.6-\mathrm{mm}$ tip diameter, $1.4-\mu \mathrm{m}$ lateral resolution, $10-\mu \mathrm{m}$ optical sectioning, and a $60-\mu \mathrm{m}$ work distance. A CellVizio Mosaic Toolbox was used to form a bigger FOV by following the probe's track. We quantified the vessel length, area, and diameter with the CellVizio Vessel Detection Module. The fiber optic probe cleaning procedure was followed in accordance with the manufacturer's instructions.

\subsection{Fixation and Clearing of Colon Samples}

After CLE imaging, the mice were deeply anesthetized and euthanized. Mice colons were removed aseptically, then washed with phosphate-buffered saline (PBS), and fixed with $4 \%$ buffered formalin for $24 \mathrm{~h}$ at $4^{\circ} \mathrm{C}$ in the dark. Fixed colons were dehydrated in methanol (Beijing Chemical Works, China) ranging from $25 \%$ to $100 \%$ (in PBS) for $3 \mathrm{~h}$ and left in $100 \%$ methanol for $24 \mathrm{~h}$ at $25^{\circ} \mathrm{C}$. Then, colons were cleared with benzyl alcohol and benzyl benzoate (BABB at a 1:2 volume ratio) solution over $24 \mathrm{~h}$ at $25^{\circ} \mathrm{C}$. $^{24}$

\subsection{Light-Sheet Fluorescence Microscopy of Mice Colons}

The cleared colons were scanned with a commercial LSFM (LaVision BioTec, Germany). We combined a magnification of $2 \times$ with a $2 \times$ objective lens (Mv PLAPO 2VC; Olympus) covered with a 6-mm working distance dipping cap. We used a supercontinuum white-light laser (SuperK EXTREME $80 \mathrm{MHz}$ VIS with wavelength from 400 to $2400 \mathrm{~nm}$; NKT Photonics, Cologne, Germany) as a laser source. The filters were set as $470 / 40 \mathrm{~nm}$ excitation and $525 / 50 \mathrm{~nm}$ emission for acriflavine and 640/30 nm excitation and 690/50 nm emission for Evans blue for the detection of cell morphology and vessels in the samples. The step size was set to $5 \mu \mathrm{m}$ and a total range of up to $2 \mathrm{~mm}$ for colon transversal scanning. The measurements were performed with 385-ms exposure times per slice, and a total imaging time of $\sim 6$ min per colon sample. Imaris software (Bitplane, Oxford Instruments Company) was used to generate $3-\mathrm{D}$ reconstructions of the tagged image file format images of the colons.

\subsection{Histological Validations}

To validate CLE and LSFM findings, mice colon sections were collected and preserved in $4 \%$ buffered formalin for histologic study. The 4- $\mu \mathrm{m}$ coronal sections of paraffin-embedded mouse colons were cut and stained with hematoxylin and eosin (H\&E) for histological scoring. ${ }^{21}$

\subsection{Statistical Analysis}

Data were represented as mean \pm standard deviation. Statistical analysis was performed in SPSS (IBM, version 23). The results of the two groups were compared using two-tail student's $t$-tests. Correlations were analyzed using Pearson's correlation coefficient (Pearson Product Moment Correlation) and significances were tested using two-tail student's $t$-tests. The differences with a $p$-value $<0.05$ were considered statistically significant. Here, $*$ denotes $p<0.05$, ** denotes $p<0.01$, and $* * *$ denotes $p<0.001$.

\section{Results}

Our multimodality imaging procedure of murine colitis models was shown in Fig. 1(a). As expected, the DSS-induced group had higher weight loss after two days than the control group [Fig. 1(b)]. Loose and bloody stools occurred three days after administration of DSS to mice. The inflammation-associated activity score was significantly higher in DSS-induced group compared to controls without DSS colitis ${ }^{21}$ [Fig. 1(c)].

\subsection{In Vivo Monitoring of Luminal Changes by MRI}

To monitor a transversal colon area for DSS-induced mice, MRI was performed at three and six days after DSS water feeding. ${ }^{25}$ A significant increased colon lumen area was observed in DSSinduced mice, as measured by T1-weighted imaging [Figs. 2(a)2(c)]. The cross-colon area was $1.27 \pm 0.61 \mathrm{~mm}^{2}$ in healthy mice and $3.0 \pm 0.61 \mathrm{~mm}^{2}(p<0.01)$ and $4.05 \pm 0.7 \mathrm{~mm}^{2}$ $(p<0.05)$ in mice with DSS colitis on day three and six, respectively [Fig. 2(d)]. 
(a)

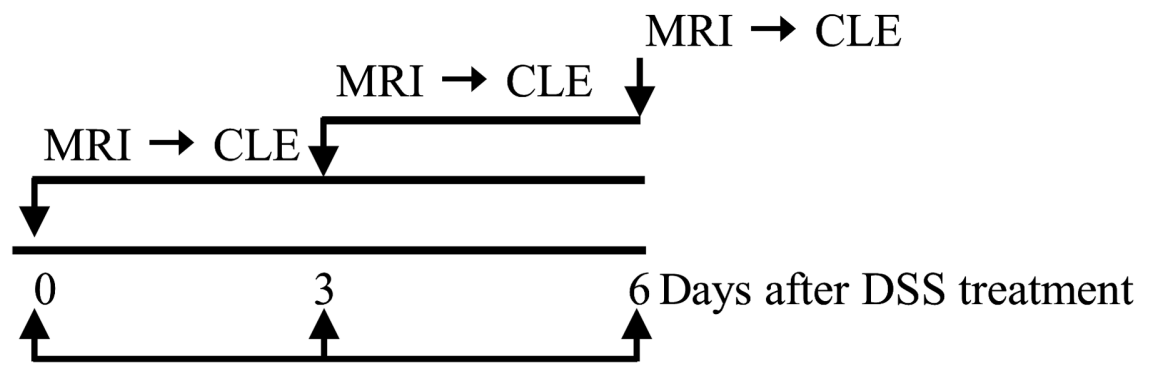

Killed for LSFM imaging

(b)

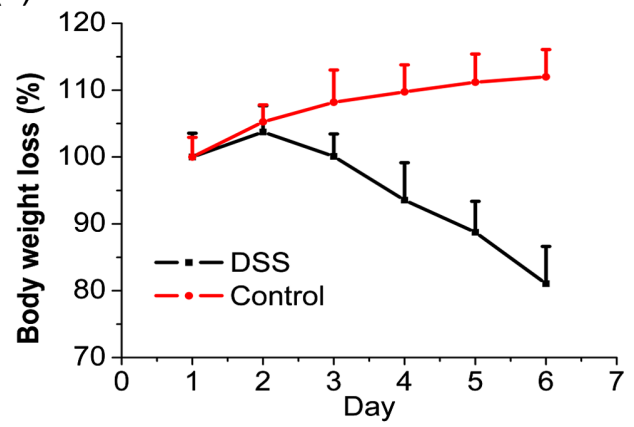

(c)

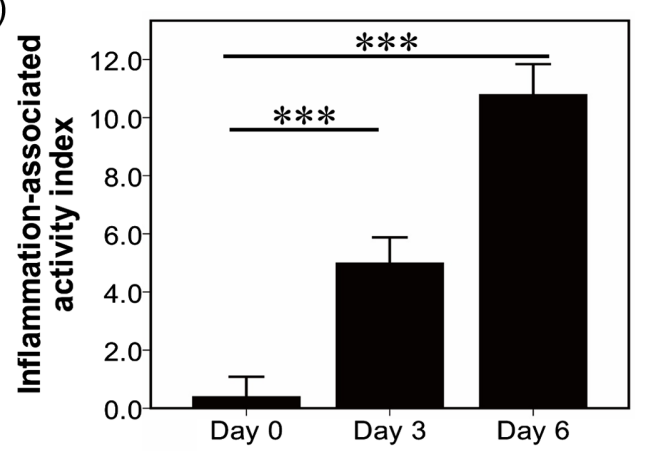

Fig. 1 (a) Schematic illustration of the DSS-induced model and the imaging procedure; (b) comparison of mice body weight loss and (c) inflammation-associated activity index score analysis.
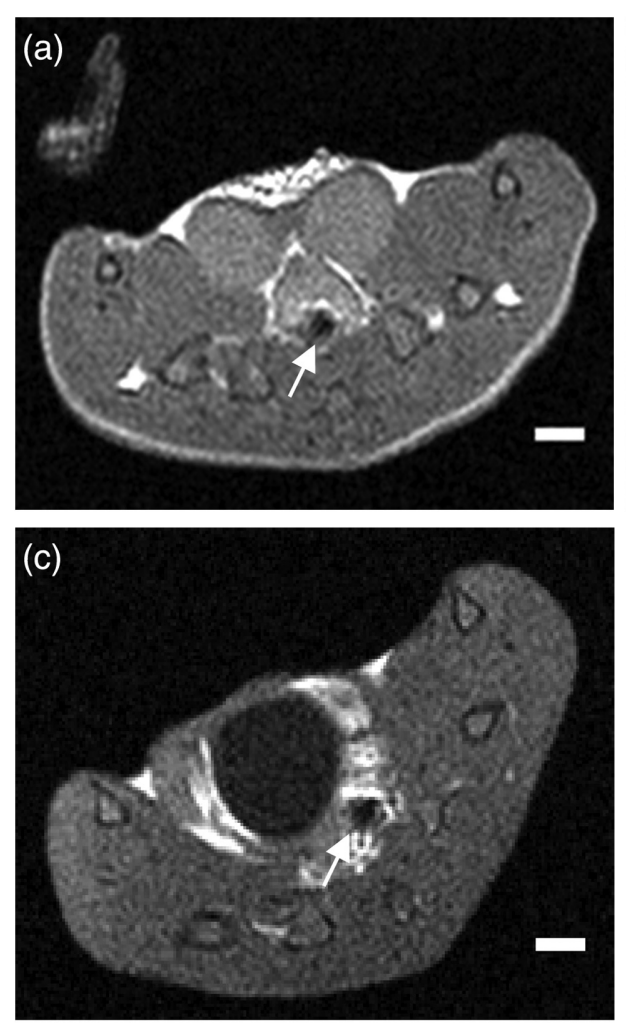

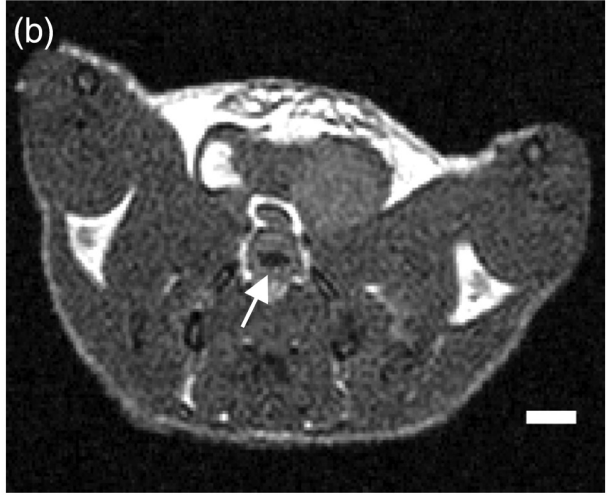

(d)

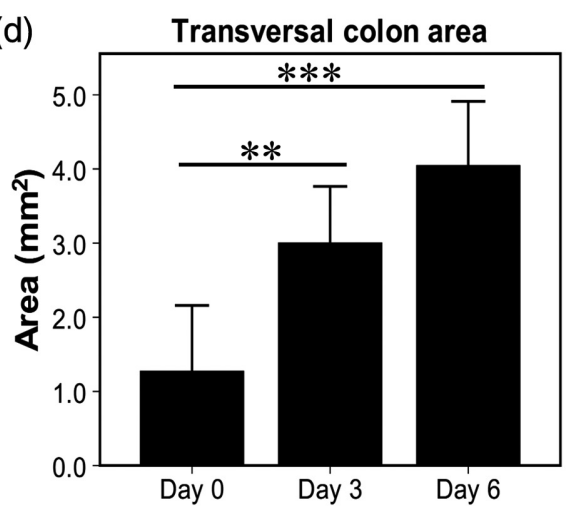

Fig. 2 T1-weighted MRI of luminal changes: (a) the colon transversal area (white arrows) for healthy mice, (b) for three days after DSS induction, and (c) for six days after DSS induction. (d) Quantification of the colon cross-sectional area for the T1-weighted images (scale bars represent $2 \mathrm{~mm})$. 

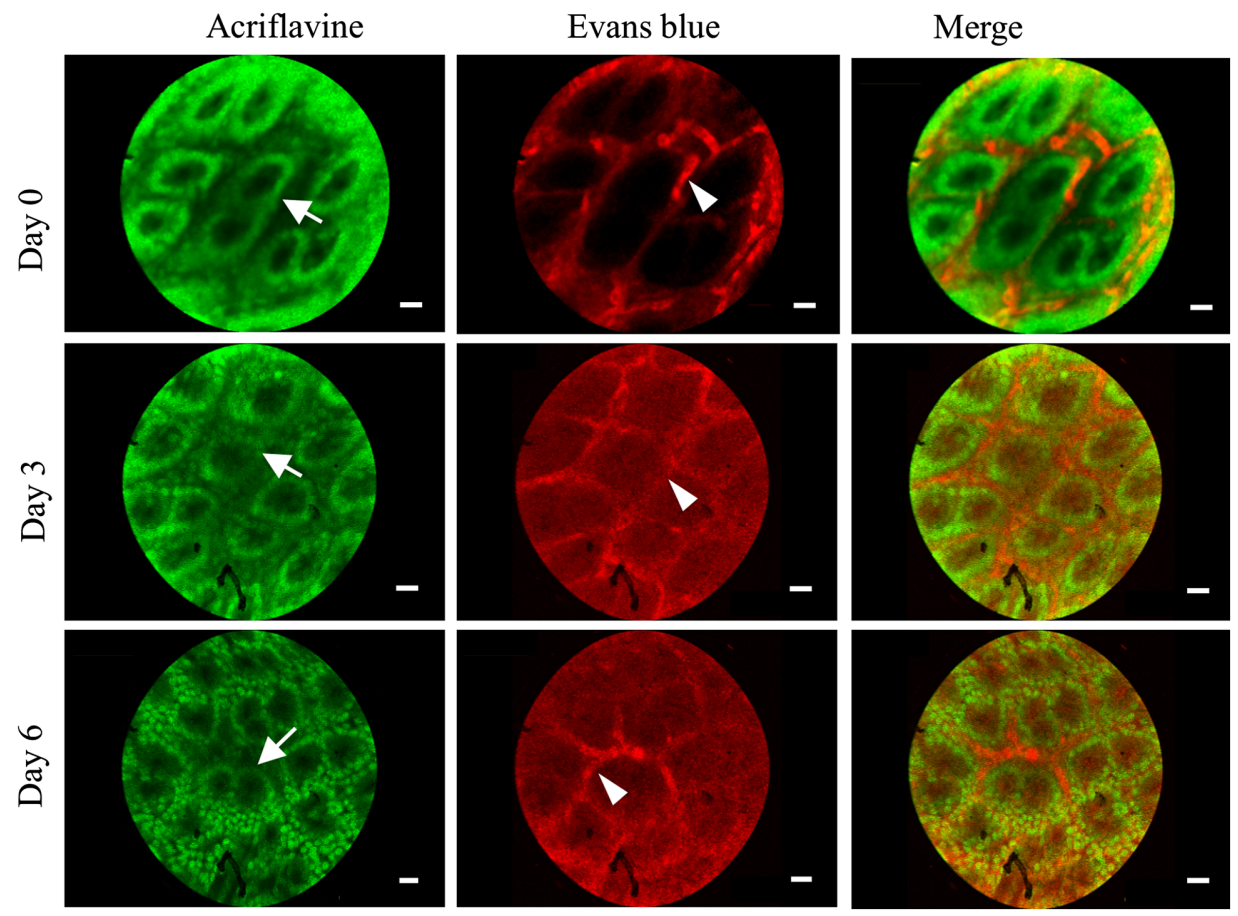

Fig. 3 Simultaneously dual-channel (488 and $660 \mathrm{~nm}$ ) CLE imaging of intestinal epithelial cells (white arrows) and vessels (white arrowheads) for the control group (Video 1, MOV, 0.8 MB [URL: https://doi .org/10.1117/1.JBO.24.1.016003.1]), three days after DSS induction (Video 2, MOV, 0.8 MB [URL: https://doi.org/10.1117/1.JBO.24.1.016003.2]), and six days after DSS induction (Video 3, MOV, 0.6 MB [URL: https://doi.org/10.1117/1.JBO.24.1.016003.3]) (scale bars are $20 \mu \mathrm{m}$ ).

Acriflavine
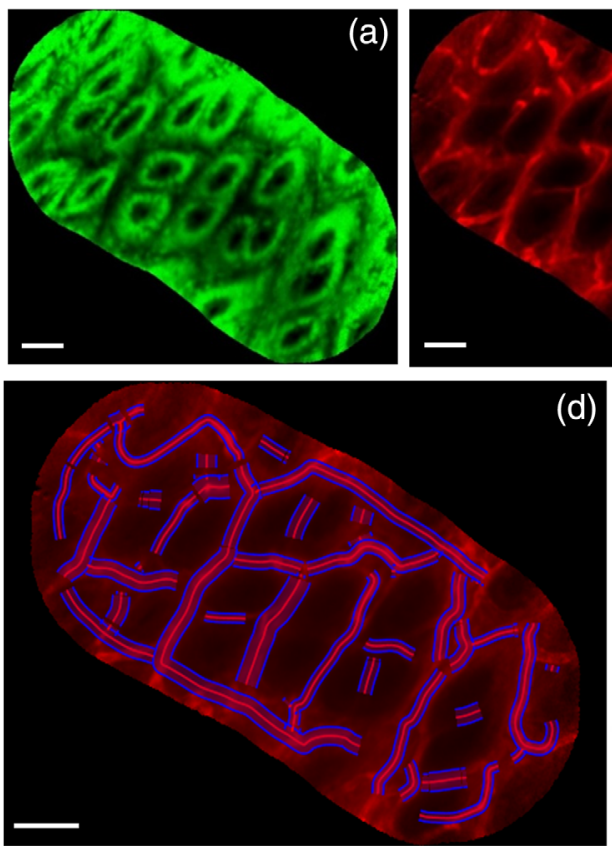

Evans blue

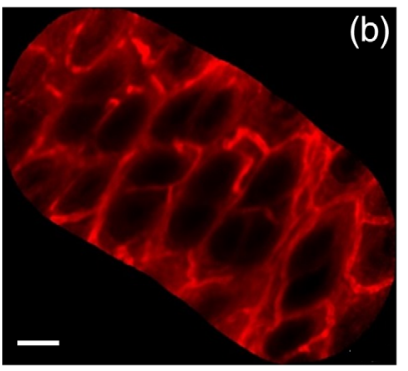

(d)

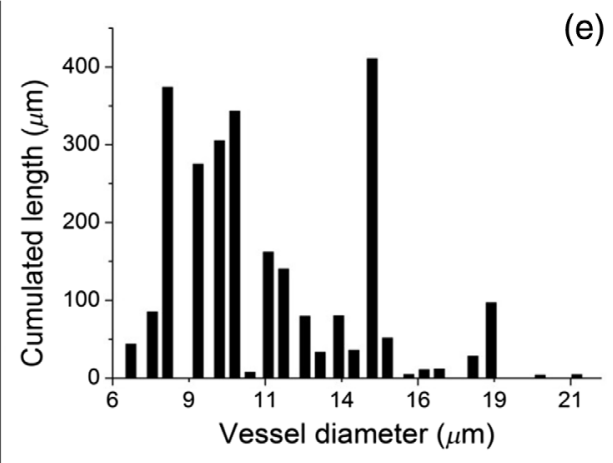

Merge

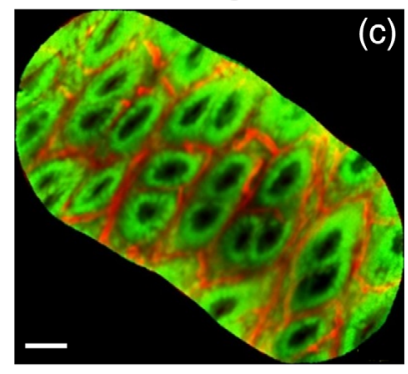

(e)

Fig. 4 CLE images stitching of fluorescently labeled epithelial cells and vessels: (a) intestinal epithelial cells and (b) vessels (Video 4, MOV, 0.5 MB [URL: https://doi.org/10.1117/1.JBO.24.1.016003.4]). (c) Merged dual-channel mosaic image. The vessels were detected under $12 \mu \mathrm{m}$ in diameter for (d) segmentation and (e) distribution analysis using CellVizio vessel detection software (scale bars are $50 \mu \mathrm{m}$ ). 


\subsection{In Vivo Monitoring of Mucosal Inflammation by CLE}

CLE gives high-resolution in vivo imaging of fluorescently stained cells and vessels throughout disease progression. We used the recently developed dual-channel CLE imaging system for monitoring intestinal epithelial cells and microvessels of colon mucosa. ${ }^{26}$ The $488-\mathrm{nm}$ laser with a fiber optic probe $0.35 \mathrm{~mm}$ in diameter allowed for visualization of the acriflavine-stained colonic epithelial cells and crypt architectures, as shown in the first column of Fig. 3. Healthy mice had intact colonic epithelial cells and crypts (Video 1). In DSS-colitis mice, we found severe cell damage and loss of crypt structure (Videos 2 and 3). The 660-nm laser excitation allowed for visualization of the mucosal vasculature architecture, blood flow, and vasculature leakage (the second column of Fig. 3). The merged results of the two-channel images are also shown in the third column of Fig. 3.

To extend the FOV of CLE, the fiber probe's trajectory was followed and the images were stitched using the mosaic technology in CellVizio [Figs. 4(a)-4(c), Video 4]. In addition, we quantitatively analyzed vessels using a vessel detection software package, which allowed for segmentation analysis of vessels, and the cumulated length, area, and diameter of the acquired images. The vessel detection analysis was $<12 \mu \mathrm{m}$ in diameter for healthy mice [Fig. 4(d)], in which the mean vessel diameter was $11.1 \mu \mathrm{m}$ and the total vessel length was $2550 \mu \mathrm{m}$.

\subsection{In-Toto 3-D Visualization of Colon Vessel Architecture and Morphology by LSFM}

LSFM has $~ 10$ times higher resolution than MRI. After MRI and CLE imaging, mice were euthanized, and the colons were removed for tissue clearing [Fig. 5(a)] by adapting the BABB protocol. ${ }^{24}$ The colonic mucosa and microvasculature were assessed in 3-D LSFM [Fig. 5(b), Video 5]. LSFM datasets showed disrupted colon villus structures and severe loss of mucosal architecture in three and six days DSS-inducted mice, respectively. We found that colon thickness and length were significantly different between healthy mice $(n=5)$ and mice with DSS-induced colitis $(n=5)$. After six days of DSS induction, we observed a significant increase in colon thickness in DSScolitis mice $[0.5 \pm 0.05 \mathrm{~mm}$ versus $0.25 \pm 0.05 \mathrm{~mm}, p<0.01$, (a)

Day 0 Day 3 Day 6

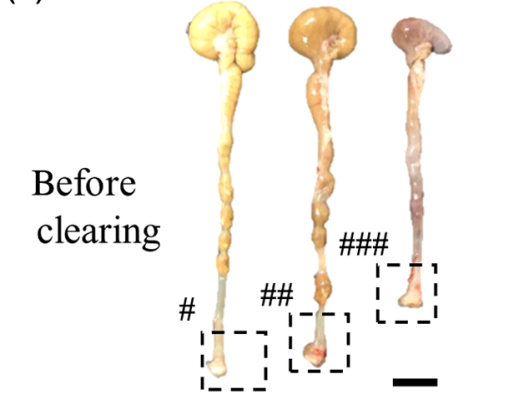

\section{After \\ \# \#\# \#\#\#}

(c)

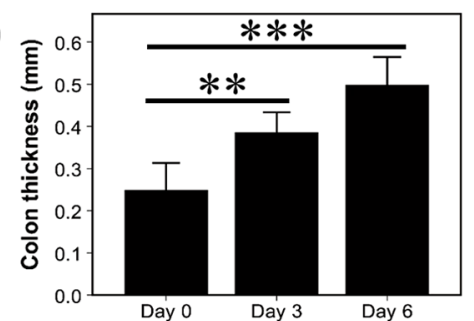

(d)

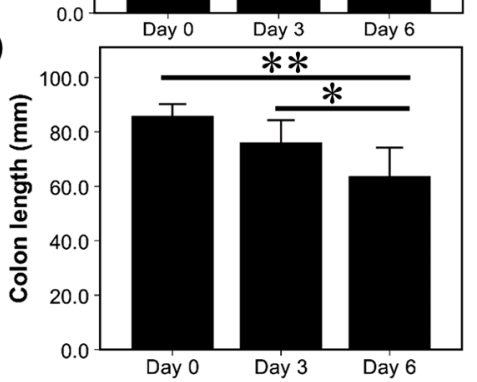

(b)
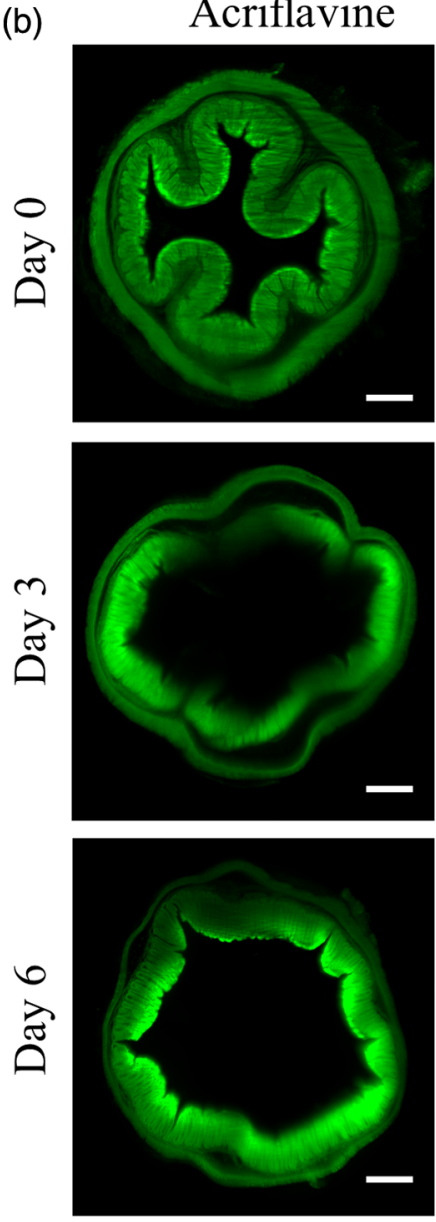

Evans blue
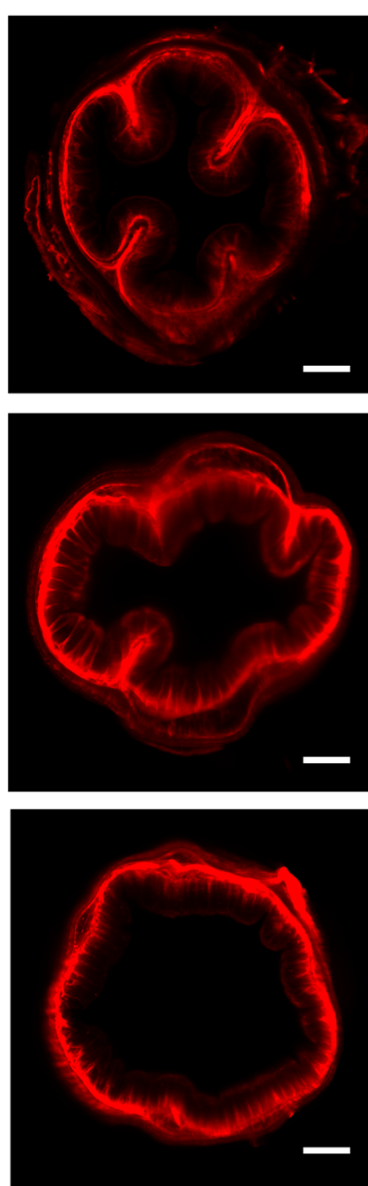

Merge
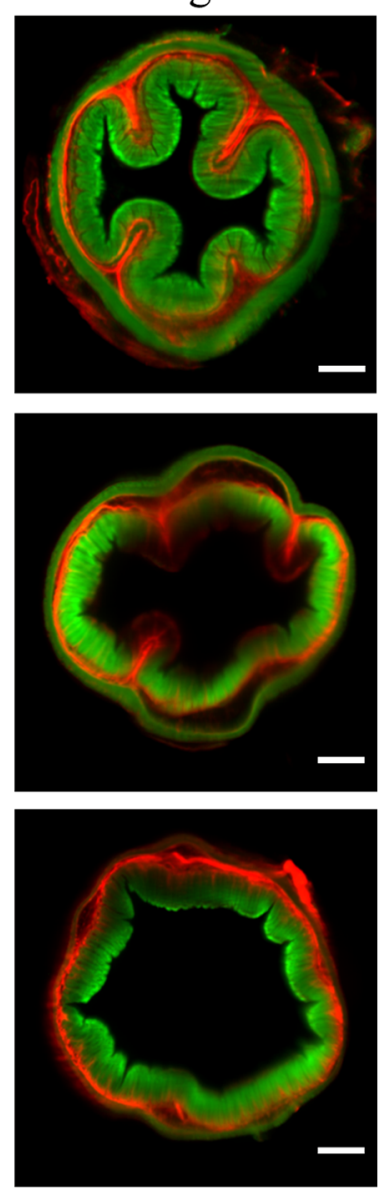

Fig. 5 LSFM imaging of fluorescently labeled epithelial cells and vessels. Illustration of the mouse colon before and after clearing by BABB protocol. (a) The distal part of a cleared colon was selected for LSFM imaging. (b) Cleared LSFM images of acriflavine- and Evans blue-stained intestinal epithelial cells (left column) and vessels (middle column) (Video 5, MOV, 2.9 MB [URL: https://doi.org/10.1117/1.JBO.24.1 $.016003 .5])$. Quantification of (c) colon thickness and (d) length. Scale bars are $1 \mathrm{~cm}$ in (a) and $50 \mu \mathrm{m}$ in (b). 
(a)

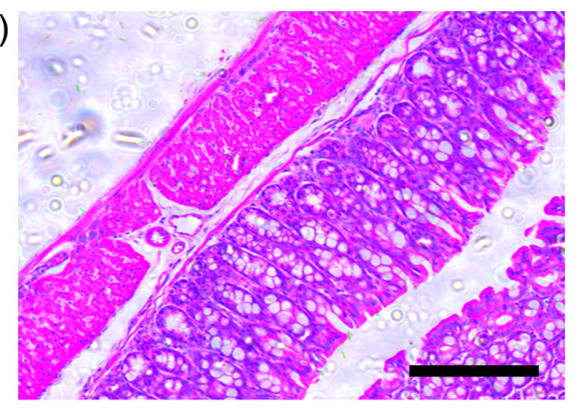

(c)

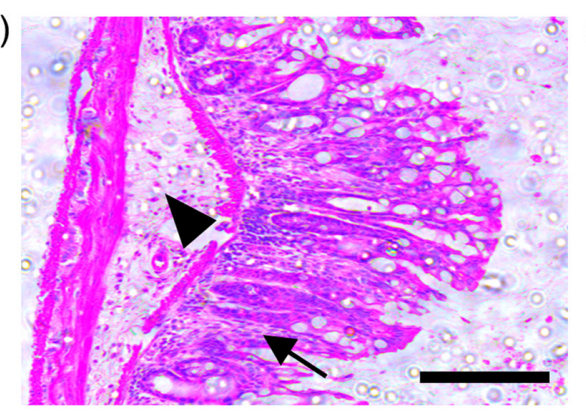

(b)

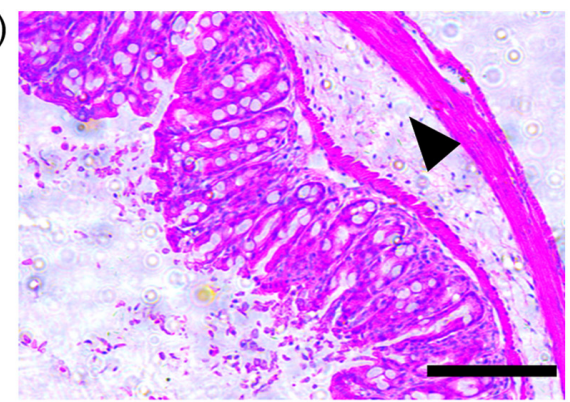

(d)

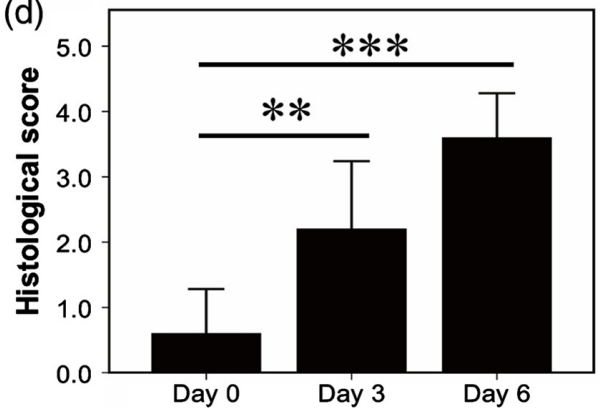

Fig. $6 \mathrm{H} \& \mathrm{E}$ staining of paraffin-embedded transversal colon sections for histological changes analysis in (a) healthy mice, (b) DSS-induced mice after three days, and (c) DSS-induced mice after six days (scale bars are $100 \mu \mathrm{m}$ ). The black arrow represents the area of severe transmural inflammation with the loss of crypt structure. Black arrowheads indicate edematous submucosal inflammatory infiltrates. (d) Histological scores of the control group and DSS-induced groups (day three and six).

Fig. 5(c)]. Colon length was $85.75 \pm 3.6 \mathrm{~mm}$ in healthy mice and $63.7 \pm 8.5 \mathrm{~mm}(p<0.05)$ in DSS-colitis mice.

\subsection{Histological Evaluation of DSS-Induced Colitis}

To characterize the development of DSS-induced colitis and validate MRI, CLE, and LSFM imaging results, histological alternations of healthy and DSS-induced mice after three and six days were assessed by H\&E staining of paraffin-embedded colon sections. As DSS-induced mucosal damage progresses, the increasing ulceration and inflammation with the loss of crypt structure were observed [Figs. 6(a)-6(c)]. A histological scoring system based on epithelial damage and inflammatory

Table 1 Correlation of the mean values from MRI (transversal areas), LSFM (colonic thickness) and CLE (the crypt architecture, microvascular alteration, and fluorescein leakage classification score) with body weight loss, colon length, histological score, and the inflammation-associated active index.

\begin{tabular}{lcccc}
\hline $\begin{array}{c}\text { Correlation } \\
\text { coefficients }\end{array}$ & $\begin{array}{c}\text { Body } \\
\text { weight } \\
\text { loss }\end{array}$ & $\begin{array}{c}\text { Colon } \\
\text { length }\end{array}$ & $\begin{array}{c}\text { Histological } \\
\text { score }\end{array}$ & $\begin{array}{c}\text { Inflammation- } \\
\text { associated } \\
\text { activity index }\end{array}$ \\
\hline MRI & $-0.69^{* *}$ & $-0.85^{* *}$ & $0.85^{* *}$ & $0.88^{* *}$ \\
LSFM & $-0.8^{* *}$ & $-0.7^{* *}$ & $0.82^{* *}$ & $0.89^{* *}$ \\
CLE & $-0.58^{*}$ & $-0.62^{*}$ & $0.79^{* *}$ & $0.82^{* *}$ \\
\hline
\end{tabular}

Note: Significance was calculated using Pearson's correlation coefficient.

${ }_{t *}^{*} p<0.05$.

${ }^{* *} p<0.01$ infiltrates was used to quantify the severity of the colitis ${ }^{21}$ [Fig. 6(d)].

\subsection{Correlation of MRI, CLE, and LSFM}

To correlate in vivo and ex vivo findings, we calculated the correlation coefficients of MRI (cross-sectional areas), LSFM (colonic thickness), and CLE (the crypt architecture, microvascular alteration and fluorescein leakage classification score $)^{6}$ results with respect to weight loss, colon length, histological score, and the inflammation-associated activity index from healthy and DSS-induced mice (after three and six days); this correlation analysis is detailed in Table 1. The MRI results had a strong correlation with weight loss, and a significant correlation with the colitis activity index, colon length, and the histological score. The 3-D LSFM results were significantly correlated with the in vivo evaluation of weight loss and the disease activity score. In addition, it was strongly correlated with colon length and the histological score determined by postmortem evaluation. The in vivo macroscopic imaging technique, MRI, was significantly correlated with the parameters representing the development and severity of colitis, and microscopic level results, observed using the LSFM imaging method, were also significantly correlated with these parameters.

\section{Discussion}

The development of optical multimodality imaging techniques can improve IBD diagnosis. ${ }^{27,28}$ However, the analysis of colon inflammation is still challenging, mainly due to heterogeneous distribution of lesions along the entire colon and in different layers of the colon. In addition, the functional and histological features of the colon are variable at different scales, even within normal colon regions. In this study, we combined in vivo MRI and CLE with ex vivo LSFM of cleared colons to assess the 
development and severity of DSS-induced colitis at different scales. First, noninvasive MRI was routinely performed to locate suspicious inflammation areas in mouse models of colitis at the macroscopic scale. Then, minimal invasive CLE was used for observing microscopic vascular architecture and cellular features alterations at these areas within mucosa. Finally, to overcome the limited FOV and imaging depth of CLE, we applied $e x$ vivo 3-D LSFM to image entire colon sections with cellular resolution. All the findings from MRI, CLE, and LSFM were cross validated with correlation analysis, which demonstrated that the correlation of these three imaging modalities was consistent.

MRI is a versatile tool and it is a noninvasive measurement of colonic transversal areas at the macroscopic level for longitudinal study. In this study, using a permanent magnet small animal MRI turned out to be feasible, safe, and low cost for routine examination of colonic areas. Moreover, in vivo MRI results were significantly correlated with ex vivo colon length and histological score. However, the resolution of MRI in our study was limited to $\sim 195 \mu \mathrm{m}$, which was unable to observe the cellular features and microvessels architecture at the microscopic level. To overcome this limitation, CLE was employed for real-time, high-resolution visualization of the epithelial cell and microvessels within the colon mucosa. For simultaneous monitoring of cell features and vessel architecture using the CLE system, we applied intravenous perfusion with acriflavine that tagged epithelial cells, followed by topical administration of Evans blue for staining the vasculature of the colon mucosal layer. After CLE, colons were cleared using BABB, a well-developed protocol. $^{24}$ The high-resolution images of clearing colon acquired by LSFM were used for 3-D reconstruction of the fluorescently tagged cells and blood vessel structure of the intact colon section. Previous studies combined white-light endoscopy with CLE to obtain high-magnification images. ${ }^{29}$ In this study, we correlated in vivo MRI at the macroscopic level with CLE at the microscopic level. In addition, we also correlated CLE in two-dimensions and LSFM in 3-D. This approach can bridge the gap between MRI and CLE at different levels, which have been separate domains in IBDs models, and it also allows for cross validation of in vivo CLE and ex vivo LSFM. Using this multimodality approach, we visualized the colon mucosal layer at the cellular level and the vessel architecture level with single vessel resolution. Furthermore, this multimodality approach not only allows in vivo visualization of inflammation within mucosa for UC study but also enables ex vivo 3-D imaging of the colon with cellular resolution for transmural investigation in $C D$, which can enhance our understanding of the pathogenesis of IBDs.

One of the main limitations of our study was CLE's relatively small FOV. As described in Sec. 1, the FOV was limited to $600 \mu \mathrm{m}$. One solution of this limitation is to stitch images acquired in a real-time scan to form a larger FOV using the mosaic image software developed by CellVizio. Another disadvantage was that clearing of the colon samples may have resulted in decreased colon size due to dehydration. This drawback may be corrected by quantifying the shrinkage of the colon volume using CT imaging before and after clearing. The LSFM images of cell and vessel parameters of cleared colons could be corrected by the volume reduction factor obtained by $\mathrm{CT}$.

In conclusion, we demonstrated the feasibility of combining in vivo MRI and CLE and ex vivo LSFM for assessing colon morphology and dynamic changes of epithelial cells and vasculature in DSS-induced colitis models. This approach showed multimodality imaging of mouse colons at different levels, which will improve the diagnostic precision of DSS colitis. This approach could also be applied to other experimental models, such as trinitrobenzene sulfonic acid colitis and oxazolone colitis. We envision that this multimodality imaging technique will improve the understanding of the pathogenesis, cell progression, and microenvironment alternation of IBDs.

\section{Disclosures}

The authors declare that this is an expanded and revised work based on our previous SPIE proceedings, and there is no conflict of interests to declare.

\section{Acknowledgments}

This paper was supported by the National Key Research and Development Program of China Nos. 2016YFC0103803, 2017YFA0700401, and 2017YFA0205200; the National Natural Science Foundation of China under Grant Nos. 81527805, 81671851, and 81227901; the Strategic Priority Research Program under Grant No. XDBS01030200, the Scientific Instrument R\&D Programs under Grant Nos. GJJSTD20170004 and YJKYYQ20170075, and the Frontier Science Key Research Program under Grant No. QYZDJ-SSW-JSC005 from the Chinese Academy of Sciences; the Beijing Municipal Science and Technology Commission under Grant No. Z161100002616022. The authors would like to acknowledge the instrumental and technical support of multimodal biomedical imaging experimental platform, Institute of Automation, Chinese Academy of Sciences.

\section{References}

1. S. Danese and C. Fiocchi, "Ulcerative colitis," N. Engl. J. Med. 365(18), 1713-1725 (2011).

2. L. J. Herrinton et al., "Incidence and mortality of colorectal adenocarcinoma in persons with inflammatory bowel disease from 1998 to 2010," Gastroenterology 143(2), 382-389 (2012).

3. L. Mielke et al., "Confocal laser endomicroscopy to monitor the colonic mucosa of mice," J. Immunol. Methods 421(6), 81-88 (2015).

4. J. Phan et al., "Time to diagnostic colonoscopy and colonoscopic findings after positive fit in an ethnically diverse cohort of us veterans," Gastroenterology 152(5), S538 (2017).

5. Y. Wu et al., "Inflammatory bowel disease: MR- and SPECT/CT-based macrophage imaging for monitoring and evaluating disease activity in experimental mouse model—pilot study," Radiology 271(2), 400-407 (2014).

6. C. Q. Li et al., "Classification of inflammation activity in ulcerative colitis by confocal laser endomicroscopy," Am. J. Gastroenterol. 105(6), 1391-1396 (2010).

7. I. Ordas et al., "Ulcerative colitis," Lancet 380(9853), 1606-1619 (2012).

8. J. Rimola et al., "Magnetic resonance imaging for evaluation of Crohn's disease: validation of parameters of severity and quantitative index of activity," Inflammatory Bowel Dis. 17(8), 1759-1768 (2011).

9. J. Walldorf et al., "In-vivo monitoring of acute DSS-colitis using colonoscopy, high resolution ultrasound and bench-top magnetic resonance imaging in mice," Eur. Radiol. 25(10), 2984-2991 (2015).

10. G. Masselli and G. Gualdi, "CT and MR enterography in evaluating small bowel diseases: when to use which modality?" Abdom. Imaging 38(2), 249-259 (2013).

11. D. N. Rasmussen et al., "Confocal laser endomicroscopy in inflammatory bowel disease-a systematic review," J. Crohn's Colitis 9(12), 1152-1159 (2015).

12. G. D. De Palma, "Confocal laser endomicroscopy in the 'in vivo' histological diagnosis of the gastrointestinal tract," World J. Gastroenterol. 15(46), 5770-5775 (2009). 
13. A. Arranz et al., "Helical optical projection tomography," Opt. Express 21(22), 25912-25925 (2013).

14. D. Dong et al., "Vertically scanned laser sheet microscopy," J. Biomed. Opt. 19(10), 106001 (2014).

15. X. Liang et al., "Stripe artifact elimination based on nonsubsampled contourlet transform for light sheet fluorescence microscopy," J. Biomed. Opt. 21(10), 106005 (2016).

16. T. Li et al., "Hybrid of two-photon microscopy and optical multimodality imaging for multi-scale imaging of small animals," SPIE BiOS 10499, 104991R (2018).

17. A. Candeo et al., "Virtual unfolding of light sheet fluorescence microscopy dataset for quantitative analysis of the mouse intestine," J. Biomed. Opt. 21(5), 056001 (2016).

18. I. Gabanyi et al., "Neuro-immune interactions drive tissue programming in intestinal macrophages," Cell 164(3), 378-391 (2016).

19. S. Melgar, A. Karlsson, and E. Michaëlsson, "Acute colitis induced by dextran sulfate sodium progresses to chronicity in C57BL/6 but not in BALB/c mice: correlation between symptoms and inflammation," $\mathrm{Am}$. J. Physiol. Gastrointest. Liver Physiol. 288(6), G1328 (2005).

20. N. Taghipour et al., "An experimental model of colitis induced by dextran sulfate sodium from acute progresses to chronicity in C57BL/6: correlation between conditions of mice and the environment," Gastroenterol. Hepatol. Bed Bench 9(1), 45-52 (2016).

21. S. Wirtz et al., "Chemically induced mouse models of acute and chronic intestinal inflammation," Nat. Protoc. 12(7), 1295-1309 (2017).

22. C. A. Schneider, W. S. Rasband, and K. W. Eliceiri, "NIH image to ImageJ: 25 years of image analysis," Nat. Methods 9(7), 671-675 (2012).

23. M. J. Waldner et al., "Confocal laser endomicroscopy and narrow-band imaging-aided endoscopy for in vivo imaging of colitis and colon cancer in mice," Nat. Protoc. 6(9), 1471-1481 (2011).

24. J. Ahnfelt-Ronne et al., "An improved method for three-dimensional reconstruction of protein expression patterns in intact mouse and chicken embryos and organs," J. Histochem. Cytochem. 55(9), 925930 (2007).

25. A. Bianchi et al., "Noninvasive longitudinal study of a magnetic resonance imaging biomarker for the quantification of colon inflammation in a mouse model of colitis," Inflammatory Bowel Dis. 22(6), 12861295 (2016)

26. G. Vargas et al., "Topical injury evaluation of the murine colorectal mucosa using confocal endomicrosopy: a valuable method for assessing mucosal injuries associated with risk of pathogen transmission," J. Microsc. 264(2), 227-237 (2016).

27. R. Atreya et al., "In vivo imaging using fluorescent antibodies to tumor necrosis factor predicts therapeutic response in Crohn's disease," Nat. Med. 20(3), 313-318 (2014).
28. E. Stanley, H. K. Moriarty, and C. G. Cronin, "Advanced multimodality imaging of inflammatory bowel disease in 2015: an update," World $J$. Radiol. 8(6), 571-580 (2016).

29. S. Coda and A. V. Thillainayagam, "State of the art in advanced endoscopic imaging for the detection and evaluation of dysplasia and early cancer of the gastrointestinal tract," Clin. Exp. Gastroenterol. 7, 133150 (2014).

Tianmeng $\mathbf{L i}$ is a second-year graduate student at the Northeastern University in Shenyang, China. Her major is biomedical engineering. She received her bachelor's degree in network engineering from Zaozhuang College, Shandong Province, China. Her current research interests include 3-D reconstruction and application of optical images.

Hui Hui is an associate professor at the Institute of Automation, Chinese Academy of Sciences, China. He received his BS and MS degrees in mechanical engineering from Chang'an University, China, in 2003 and 2006, respectively, and his PhD in mechanical engineering from the University of Franche-Comté, France, in 2013. His current research interests include multimodal molecular imaging and optical microscopy.

Chaoen $\mathrm{Hu}$ is a research assistant at the Institute of Automation, Chinese Academy of Sciences, China. He received his master's degree in control engineering major of Harbin University of Science and Technology, China. His current research interests include image processing and visualization.

$\mathrm{He} \mathrm{Ma}$ is an associate professor, vice dean, master tutor at the Institute of Biomedical and Information Engineering, Northeastern University. He received his $\mathrm{PhD}$ in computer science and technology from the Department of Computer Science, National University of Singapore in 2014. His current research interests include lung cancer imagery and medical image process.

Xin Yang is a professor at the Institute of Automation, Chinese Academy of Sciences, China. She received her $\mathrm{PhD}$ in precision instruments and optoelectronics engineering from Tianjin University, China, in 2000. Her current research interests include pattern recognition and medical image process.

Jie Tian is a professor at the Institute of Automation, Chinese Academy of Sciences, China. He received his PhD in artificial intelligence from the Institute of Automation, Chinese Academy of Sciences, China, in 1992. His current research interests include medical image process and molecular imaging. 